\title{
QUANTITATIVE ESTIMATES FOR SOME MODIFIED BERNSTEIN-STANCU OPERATORS
}

\author{
VOICHIŢA ADRIANA RADU
}

Received 17 February, 2015

Abstract. In the papers [11,12] starting with the Bernstein operators, some Stancu type operators are constructed

$$
\begin{gathered}
C_{n}: Y \rightarrow \Pi_{n} \\
\left(C_{n} f\right)(x)=\sum_{k=0}^{n} \frac{k !}{n^{k}}\left(\begin{array}{l}
n \\
k
\end{array}\right) \mathbf{m}_{k, n}\left[0, \frac{1}{n}, \ldots, \frac{k}{n} ; f\right] x^{k}, f \in C[0,1],
\end{gathered}
$$

where $\mathrm{Y}$ is the linear space of all functions $[0,1] \rightarrow \mathbb{R}$ and the real numbers $\left(m_{k, n}\right)_{k=0}^{\infty}$ are selected in order to preserve some important properties of Bernstein operators.

For $\mathbf{m}_{j, n}=\frac{\left(a_{n}\right)_{j}}{j !}, a_{n} \in(0,1]$ we obtained Bernstein-Stancu operators

$$
\left(\bar{C}_{n} f\right)(x)=\sum_{k=0}^{n} \frac{\left(a_{n}\right)_{k}}{n^{k}}\left(\begin{array}{l}
n \\
k
\end{array}\right)\left[0, \frac{1}{n}, \ldots, \frac{k}{n} ; f\right] x^{k} .
$$

The aim of this paper is to give some estimates for this operators using moduli of smoothness of first and second order.

2010 Mathematics Subject Classification: 41A36; 41A10; 41A25

Keywords: approximation by positive linear operators, Bernstein operators, Stancu operators, Bernstein-Stancu operators, the second order modulus of smoothness

\section{INTRODUCTION}

First of all, we recall some notions and operators which will be used in the paper.

Let $\Pi$ be the algebra of polynomials with real coefficients and $\Pi_{n}$ be the linear space of all real polynomials of degree $\leq n$.

For $k \in \mathbb{N}, z \in \mathbb{C}$ let $(z)_{0}=1$ and $(z)_{k}=z(z+1) \ldots(z+k-1)$.

For $n \in \mathbb{N}$, let $B_{n}: Y \rightarrow \Pi_{n}$ be the Bernstein operators, defined for any $f \in C[0,1]$ by

$$
\left(B_{n} f\right)(x)=\sum_{k=0}^{n} b_{n, k}(x) f\left(\frac{k}{n}\right)
$$

(c) 2018 Miskolc University Press 
where

$$
b_{n, k}(x)=\left(\begin{array}{l}
n \\
k
\end{array}\right) x^{k}(1-x)^{n-k}
$$

are the Bernstein fundamental polynomials.

For $g:[0,1] \rightarrow \mathbb{R}$ the Stancu operators $S_{k}^{<b>}: g \rightarrow S_{k}^{<b>} g, k \in \mathbb{N}$ are defined as $\left(S_{0}^{<b>} g\right)(x)=g(0)$ and for $k \in\{1,2, \ldots\}$

$$
\left(S_{k}^{<b>} g\right)(x)=\frac{1}{b_{k}} \sum_{j=0}^{k}\left(\begin{array}{l}
k \\
j
\end{array}\right)(b x)_{j}(b-b x)_{k-j} \cdot g\left(\frac{j}{k}\right), x \in[0,1]
$$

where $b \in[0,1]$ is a real parameter (see $[13,24,25])$.

\section{THE CONSTRUCTION OF THE MODIFIED BERNSTEIN-STANCU OPERATORS}

Approximation theory has been used in the theory of approximation of continuous functions by means of sequences of positive linear operators and still there remains a very active area of research. There are many approximating operators that their Korovkin type approximation properties and rates of convergence are investigated.

We list some of the mathematicians that relate their names to this fields of constructing and studding approximation properties of the linear and positive operators: A. Lupaş [20], O. Agratini [5], D. Bărbosu [8], [9], I. Gavrea, H.H. Gonska and D.P. Kacso [16], [17], O. Dogru [14], U. Abel, M. Ivan, R. Păltănea [1] and Y. Kageyama [18].

A new direction of generalization of the linear and positive operators are q-calculus as we can see in the pioneering works of A. Lupaş [19] and G.M. Philips [23]. Some of the most recent appearances in this direction are the papers of O. Agratini [6], P.N. Agrawal, Z. Finta and A. Sathish Kumar [15], [7], G. Nowak and V. Gupta [21], A.M. Acu, C.V. Muraru, D. Bărbosu and D.F. Sofonea [2], [4] and [3].

In [11] was constructed a new class of linear and positive operators starting with the derivatives of the Bernstein operators.

For $j \in\{0,1, \ldots, n\}$

$$
\frac{1}{j !} \frac{d^{j}\left(B_{n} f\right)(x)}{d x^{j}}=\left(\begin{array}{l}
n \\
j
\end{array}\right) \frac{j !}{n^{j}} \sum_{k=0}^{n-j} b_{n-j, k}(x)\left[\frac{k}{n}, \frac{k+1}{n}, \ldots, \frac{k+j}{n} ; f\right],
$$

the following formula holds

$$
\left(B_{n} f\right)(x)=\sum_{k=0}^{n} \frac{k !}{n^{k}}\left(\begin{array}{l}
n \\
k
\end{array}\right)\left[0, \frac{1}{n}, \ldots, \frac{k}{n} ; f\right] x^{k}
$$


Starting with (2.1), we investigated the modifications

$$
\begin{gathered}
C_{n}: Y \rightarrow \Pi_{n} \\
\left(C_{n} f\right)(x)=\sum_{k=0}^{n} \frac{k !}{n^{k}}\left(\begin{array}{l}
n \\
k
\end{array}\right) \mathbf{m}_{k, n}\left[0, \frac{1}{n}, \ldots, \frac{k}{n} ; f\right] x^{k}, f \in Y,
\end{gathered}
$$

where the real numbers $\left(m_{k, n}\right)_{k=0}^{\infty}$ are selected in order to preserve some important properties of Bernstein operators.

Observe that from (2.2)

$$
\left\{\begin{array}{l}
C_{n} e_{0}=\mathbf{m}_{0, n} \\
C_{n} e_{1}=\mathbf{m}_{1, n} e_{1} \\
C_{n} e_{2}=\mathbf{m}_{2, n} e_{2}+\frac{e_{1}}{n}\left(\mathbf{m}_{1, n}-\mathbf{m}_{2, n} e_{1}\right) \\
\left(C_{n} \Omega_{2, x}\right)(x)=\left(\mathbf{m}_{2, n}-2 \mathbf{m}_{1, n}+\mathbf{m}_{0, n}\right) x^{2}+\frac{x}{n}\left(\mathbf{m}_{1, n}-\mathbf{m}_{2, n} x\right)
\end{array}\right.
$$

where $e_{j}(t)=t^{j}$ and $\Omega_{2, x}=(t-x)^{2}$.

In the following we shall consider that $\mathbf{m}_{0, n}=1$ and $\lim _{n \rightarrow \infty} \mathbf{m}_{1, n}=1$.

We also consider that

$$
\mathbf{m}_{j, n}=\frac{\left(a_{n}\right)_{j}}{j !}, a_{n} \in(0,1]
$$

Then the operator $C_{n}$ from (2.2), denoted further by $\bar{C}_{n}$, becomes

$$
\left(\bar{C}_{n} f\right)(x)=\sum_{k=0}^{n} \frac{\left(a_{n}\right)_{k}}{n^{k}}\left(\begin{array}{l}
n \\
k
\end{array}\right)\left[0, \frac{1}{n}, \ldots, \frac{k}{n} ; f\right] x^{k}, f \in Y .
$$

$\bar{C}_{n}$ are called Bernstein-Stancu operators, when $a_{n} \in(0,1)$ (see [11], Definition $11)$.

\section{A SUMMING UP OF THE APPROXIMATION PROPERTIES OF $\bar{C}_{n}$ OPERATOR}

First, in [12], it is demonstrated that $\bar{C}_{n}$ is a linear and positive operator that transform any polynomial of degree $s \leq n$ into a polynomial of degree $s$ and preserves the convexity of order $j$, if $j, n \in \mathbb{N}^{*}, 0 \leq j \leq n-2$.

Also, the operator $\bar{C}_{n}$ from (2.3) may be written in the Bernstein basis in the form ([11], Theorem 10)

$$
\left(\bar{C}_{n} f\right)(x)=\sum_{k=0}^{n} b_{n, k}(x) \bar{C}_{k, n}[f]
$$

with

$$
\bar{C}_{k, n}[f]=\frac{1}{k !} \sum_{j=0}^{k}\left(\begin{array}{l}
k \\
j
\end{array}\right) f\left(\frac{j}{n}\right)\left(a_{n}\right)_{j}\left(1-a_{n}\right)_{k-j} .
$$


In the same time $\bar{C}_{n}$ can be written using Stancu functionals.

Observe that

$$
\bar{C}_{0} f=\bar{C}_{0,0}[f]:=f(0)
$$

and

$$
\left(S_{k}^{<1>} g\right)\left(a_{n}\right)=\frac{1}{k !} \sum_{j=0}^{k}\left(\begin{array}{l}
k \\
j
\end{array}\right)\left(a_{n}\right)_{j}\left(1-a_{n}\right)_{k-j} g\left(\frac{j}{k} \cdot \frac{k}{n}\right), k \geq 1 .
$$

Therefore,

$$
\bar{C}_{k, n}[f]=\left(S_{k}^{<1>} g_{n, k}^{<f>}\right)\left(a_{n}\right)
$$

with

$$
g_{n, k}^{<f>}(t)=f\left(t \frac{k}{n}\right), k \geq 1 .
$$

In order to provide the convergence theorem, the following identities hold true ([11], Lemma 8):

$$
\left\{\begin{array}{l}
\left(\overline{C_{n}} e_{0}\right)(x)=1 \\
\left(\overline{C_{n}} e_{1}\right)(x)=a_{n} x=x-\left(1-a_{n}\right) x \\
\left(\overline{C_{n}} e_{2}\right)(x)=x^{2}+\frac{x(1-x)}{n} a_{n}+\frac{1-a_{n}}{2}\left(\frac{a_{n}}{n}-\left(2+a_{n}\right)\right) x^{2} .
\end{array}\right.
$$

Also, if $f \in Y, \Omega_{2, x}=(t-x)^{2}$ and $a_{n} \in(0,1]$, then

$$
\left(\overline{C_{n}} \Omega_{2, x}\right)(x)=\frac{x(1-x)}{n} a_{n}+x^{2}\left(1-a_{n}\right)\left(\frac{2-a_{n}}{2}+\frac{a_{n}}{2 n}\right) .
$$

Moreover

$$
\left|\left(\overline{C_{n}} \Omega_{2, x}\right)(x)\right| \leq \frac{a_{n}}{4 n}+\left(1-a_{n}\right), \quad \forall x \in[0,1] .
$$

Applying the Bohman - Korovkin theorem and the above assertions ([12], Theorem 5) we can see that the sequence $\left\{\bar{C}_{n} f\right\}_{n \geq 1}$ converges to f, uniformly on $[0,1]$ for any $f \in Y$.

The asymptotic behavior of the sequence $\left(\bar{C}_{n}\right)_{n=1}^{\infty}$ on a certain subspaces of $C[-1,1]$ is given in the following proposition ([11], Theorem 15) and it was demonstrated applying a version of a general proposition given by R. G. Mamedov:

Theorem 1 ([11], Theorem 15). Suppose $x_{0} \in[0,1]$ and $f^{\prime \prime}\left(x_{0}\right)$ exists. If $a_{n} \in$ $(0,1), \lim _{n \rightarrow \infty} a_{n}=1$ and exists $L:=\lim _{n \rightarrow \infty} n\left(1-a_{n}\right)$, then

$\lim _{n \rightarrow \infty} n\left[f\left(x_{0}\right)-\left(\bar{C}_{n} f\right)\left(x_{0}\right)\right]=-\frac{x(1-x)}{2} f^{\prime \prime}\left(x_{0}\right)+\left[x_{0} f^{\prime}\left(x_{0}\right)-\frac{x_{0}^{2}}{4} f^{\prime \prime}\left(x_{0}\right)\right] L$.

In order to obtain an overview of the approximation properties of this ope- rator, we add here some more properties. 
Lemma 1. If $f \in C[0,1]$, then $\left\|\bar{C}_{n} f\right\| \leq\|f\|$, where $\|\cdot\|$ is the uniform norm on $C[0,1]$.

Proof. Using the fact that the operator is linear and positive we have the identity

$$
\left\|\bar{C}_{n}\right\|=\left\|\bar{C}_{n} e_{0}\right\| \text {. }
$$

And from the above property $\left(\overline{C_{n}} e_{0}\right)(x)=e_{0}$ then $\left\|\bar{C}_{n}\right\|=1$.

Lemma 2. The operator $\bar{C}_{n} f$ can be represented by the following expansion

$$
\left(\bar{C}_{n} f\right)(x)=f(0)+\sum_{k=1}^{n} \frac{\left(a_{n}\right)_{k} x^{k}}{k !}\left(\begin{array}{l}
n \\
k
\end{array}\right)\left(\Delta_{n^{-1}}^{k} f\right)(0),
$$

where $\Delta_{n^{-1}}^{k} f(0)$ is the finite difference of order $k$, with the step $n^{-1}$ and the starting point 0 of the function $f$, that is

$$
\Delta_{n^{-1}}^{k} f(0)=\sum_{j=0}^{k}(-1)^{j}\left(\begin{array}{l}
k \\
j
\end{array}\right) f\left(\frac{k-j}{n}\right) .
$$

Proof. By making use of the following relation between divided differences and finite differences

$$
\left[x_{0}, x_{0}+h, \ldots, x_{0}+k h ; f\right]=\frac{1}{k ! \cdot h^{k}} \cdot \Delta_{h}^{k} f\left(x_{0}\right)
$$

we obtain

$$
\left[0, \frac{1}{n}, \ldots, \frac{k}{n} ; f\right]=\frac{n^{k}}{k !} \Delta_{n^{-1}}^{k} f(0) .
$$

Replacing it in (2.3) we are led to the desired formula.

Remark 1. If we set $x=0$ in the expansion formula from above, then we find

$$
\left(\bar{C}_{n} f\right)(0)=f(0) \text {. }
$$

Naturally follows the fact that the polynomial (2.3) is interpolating at the end 0 of the interval $[0,1]$ i.e. $\left(\bar{C}_{n} f\right)(0)=f(0)$.

\section{ESTIMATES FOR $\bar{C}_{n}$ IN TERMS OF MODULi OF SMOOTHNESS}

The main tools to estimating the degree of approximation by positive linear functionals and operators are the moduli of smoothness of first and second order, given by (see, for example, [10])

$$
\begin{gathered}
\omega(f ; \delta):=\sup \{|f(x)-f(t)|: x, t \in I,|x-t| \leq \delta\} \\
\omega_{2}(f ; \delta):=\sup \left\{\left|f(x)-2 f\left(\frac{x+t}{2}\right)+f(t)\right|: x, t \in I,|x-t| \leq 2 \delta\right\}
\end{gathered}
$$


for $f \in C(I)$ and $\delta \geq 0$.

For $f \in C[a, b]$, a useful modification represents the least concave majorant of $\omega(f ; \cdot)$ given by

$$
\tilde{\omega}(f ; \epsilon)=\left\{\begin{array}{ccc}
\sup _{0 \leq x \leq \epsilon \leq y \leq b-a, x \neq y} \frac{(\epsilon-x) \omega(f, y)+(y-\epsilon) \omega(f, x)}{y-x} & \text { if } & 0 \leq \epsilon \leq b-a \\
\tilde{\omega}(f ; \epsilon)=\omega(f ; b-a) & \text { if } \quad \epsilon>b-a
\end{array}\right.
$$

First, using the result obtained by O. Shisha and B. Mond in 1968, we recall from [12] the estimation for $\overline{C_{n}}$ using the modulus of continuity.

Theorem 2 ([12], Theorem 9). If $a_{n} \in(0,1)$ such that $\lim _{n \rightarrow \infty} a_{n}=1$ and $L:=$ $\lim _{n \rightarrow \infty} n\left(1-a_{n}\right)$ exists, then for any $f \in Y, x \in[0,1]$ and $n \geq 1$, the Bernstein-Stancu operators (2.3) verify

$$
\left|\left(\overline{C_{n}} f\right)(x)-f(x)\right| \leq\left(1+\frac{1}{\delta} \sqrt{\frac{a_{n}}{4 n}+\left(1-a_{n}\right)}\right) \omega(f ; \delta) .
$$

Corollary 1 ([12], Corollary 10). If $a_{n} \in(0,1)$ such that $\lim _{n \rightarrow \infty} a_{n}=1$ and $L:=$ $\lim _{n \rightarrow \infty} n\left(1-a_{n}\right)$ exists, then for any $f \in Y, x \in[0,1]$ and $n \geq 1$, the Bernstein-Stancu operators (2.3) verify

$$
\left|\left(\overline{C_{n}} f\right)(x)-f(x)\right| \leq\left(1+\sqrt{\frac{a_{n}}{4}+n\left(1-a_{n}\right)}\right) \omega\left(f ; \frac{1}{\sqrt{n}}\right) .
$$

Corollary 2 ([12], Corollary 11). If $a_{n} \in(0,1)$ such that $\lim _{n \rightarrow \infty} a_{n}=1$ and $L:=$ $\lim _{n \rightarrow \infty} n\left(1-a_{n}\right)$ exists, then for any $f \in Y, x \in[0,1], n \geq 1$ and

$$
\delta=\sqrt{\frac{a_{n}}{4 n}+\left(1-a_{n}\right)}
$$

then Bernstein-Stancu operators verify

$$
\left|\left(\overline{C_{n}} f\right)(x)-f(x)\right| \leq 2 \omega(f ; \delta) .
$$

Theorem 3 ([12], Theorem 12). If $a_{n} \in(0,1)$ such that $\lim _{n \rightarrow \infty} a_{n}=1$ and $L:=$ $\lim _{n \rightarrow \infty} n\left(1-a_{n}\right)$ exists, then for any $f \in Y, x \in[0,1]$ and $n \geq 1$, the Bernstein-Stancu operators (2.3) verify

$$
\left|\left(\overline{C_{n}} f\right)(x)-f(x)\right| \leq\left|a_{n} x-x\right| \cdot\left|f^{\prime}(x)\right|+2 \delta \omega\left(f^{\prime} ; \delta\right),
$$

where

$$
\delta=\sqrt{\frac{a_{n}}{4 n}+\left(1-a_{n}\right)} .
$$


The main results of this paper are direct estimates via $\omega, \tilde{\omega}$ and $\omega_{2}$.

Let $K=[a, b]$ and $K^{\prime} \subseteq K$ be also compact and let $L: C(K) \rightarrow C\left(K^{\prime}\right)$ be a positive linear operator. The following result was obtain by H.H. Gonska in [17].

Theorem 4. For the linear and positive operator $L$ that reproduce the constant functions, the following inequality holds:

$$
|L(f ; x)-f(x)| \leq \max \left\{1, \frac{1}{h} \cdot L\left(\left|e_{1}-x\right| ; x\right)\right\} \cdot \tilde{\omega}(f ; h),
$$

for all $f \in C(K), x \in K^{\prime}$ and $h>0$.

In our case, we have

Theorem 5. If $a_{n} \in(0,1)$ such that $\lim _{n \rightarrow \infty} a_{n}=1$ and $L:=\lim _{n \rightarrow \infty} n\left(1-a_{n}\right)$ exists, then for any $f \in Y, x \in[0,1]$ and $n \geq 1$, for Bernstein-Stancu operators (2.3), the following inequality holds:

$$
\left|\left(\overline{C_{n}} f\right)(x)-f(x)\right| \leq \tilde{\omega}\left(f ; \sqrt{\frac{a_{n}}{4 n}+\left(1-a_{n}\right)}\right) .
$$

Proof. Using the Gonska's result and the inequality (3.1) we obtain

$$
\left|\left(\overline{C_{n}} f\right)(x)-f(x)\right| \leq \max \left\{1, \frac{1}{h} \cdot \sqrt{\frac{a_{n}}{4 n}+\left(1-a_{n}\right)}\right\} \cdot \tilde{\omega}(f ; h),
$$

and putting $h=\sqrt{\frac{a_{n}}{4 n}+\left(1-a_{n}\right)}$ leads to the desired result.

Due to the fact that $\omega_{2}$ annihilates linear functions, it is advantageous to measure the degree of approximation by means of this modulus of smoothness.

Further, we recall the following results given by R. Păltănea in [22].

Theorem 6. For any $f \in C(K)$ all $x \in C\left(K^{\prime}\right)$ and $0<h<\frac{1}{2}$ length $(K)$ we have

$$
\begin{aligned}
\mid L(f ; x)- & f(x)|\leq| L\left(e_{0} ; x\right)-1|\cdot| f(x)|+| L\left(e_{1}-x ; x\right) \mid \cdot \frac{1}{h} \omega_{1}(f, h) \\
& +\left(L\left(e_{0} ; x\right)+\frac{1}{2} \cdot \frac{1}{h^{2}} L\left(\left(e_{1}-x\right)^{2} ; x\right) \omega_{2}(f, h) .\right.
\end{aligned}
$$

Thus we can state

Theorem 7. If $a_{n} \in(0,1)$ such that $\lim _{n \rightarrow \infty} a_{n}=1$ and $L:=\lim _{n \rightarrow \infty} n\left(1-a_{n}\right)$ exists, then for any $f \in Y, x \in[0,1], n \geq 1$ and $0<h \leq \frac{1}{2}$ we have:

$$
\left|\left(\overline{C_{n}} f\right)(x)-f(x)\right| \leq \frac{1}{h}\left(1-a_{n}\right) \omega(f, h)+\left(1+\frac{1}{2} \cdot \frac{1}{h^{2}}\left(\frac{a_{n}}{4 n}+\left(1-a_{n}\right)\right)\right) \omega_{2}(f, h)
$$


Proof. The assertion follows from the Păltănea's theorem using inequality (3.1).

\section{REFERENCES}

[1] U. Abel, M. Ivan, and R. Paltanea, "The durrmeyer variant of an operator defined by d.d. stancu," Applied Mathematics and Computation, vol. 259, pp. 116-123, 2015.

[2] A. Acu, "Stancu-schurer-kantorovich operators based on q-integers," Applied Mathematics and Computation, vol. 259, pp. 896-907, 2015.

[3] A. Acu, D. Barbosu, and D. Sofonea, "Note on a q-analogue of stancu-kantorovich operators," Miskolc Mathematical Notes, vol. 16, no. 1, pp. 3-15, 2015.

[4] A. Acu and C. Muraru, "Approximation properties of bivariate extension of q-bernstein-schurerkantorovich operators," Results in Mathematics, vol. 67, no. 3, pp. 265-279, 2015.

[5] O. Agratini, "Linear combinations of d.d. stancu polynomials," Rev. D'Analyse Num. et Th. de l'Approx., vol. XXVII, no. 1, pp. 15-22, 1998.

[6] O. Agratini, "On a q-analogue of stancu operators," Central European Journal of Mathematics, vol. 8, no. 1, pp. 191-198, 2010.

[7] P. Agrawal, Z. Finta, and A. Sathish Kumar, "Bernstein-schurer-kantorovich operators based on q-integers," Applied Mathematics and Computation, vol. 256, pp. 222-231, 2015.

[8] D. Barbosu, "Scurer-stancu type operators," Studia Univ. Babeş-Bolyai Math., vol. XLVIII, no. 3, pp. 31-35, 2003.

[9] D. Barbosu, "Durrmeyer-schurer type operators," Facta Universitatis (Nis), Ser. Math. Inform., vol. 19, pp. 65-72, 2004.

[10] V. Cleciu, "On some classes of bernstein type operators which preserve the global smoothness in the case of univariate functions," Acta Univ. Apulensis Math. Inform., vol. 6, pp. 91-100, 2003.

[11] V. Cleciu, "Approximation properties of a class of bernstein-stancu type operators," in Proceedings of the international conference NAAT, O. Agratini, Ed. Cluj-Napoca: Casa Cărţii de Ştiinţă, 2006, pp. 171-178.

[12] V. Cleciu, "Bernstein-stancu operators," Studia Univ. Babeş-Bolyai Math., vol. LII, no. 4, pp. 53-65, 2007.

[13] B. Della Vechia, "On the approximation of functions by means of the operators of d.d. stancu," Studia Univ. Babeş-Bolyai Math., vol. XXXVII, pp. 3-36, 1992.

[14] O. Dogru, "Approximation properties of a generalization of positive linear operators," J. Math. Anal. Appl., vol. 342, pp. 161-170, 2008.

[15] Z. Finta, "Direct and converse results for q-bernstein operators," in Proceedings of the Edinburgh Mathematical Society, no. 52, United Kingdom, 2009, pp. 339-349.

[16] I. Gavrea, H. Gonska, and D. Kacso, "Positive linear operators with equidistant nodes," Comput.Math.Appl., vol. 8, pp. 23-32, 1996.

[17] H. Gonska, "On approximation by linear operators: improved estimates," Anal.Numer.Theor.Approx., vol. 14, pp. 7-32, 1985.

[18] Y. Kageyama, "A new class of modified bernstein operators," J.of App. Theory, vol. 101, pp. 121147, 1999.

[19] A. Lupas, "A q-analogue of the bernstein operator," Seminar on Numerical and Statistical Calculus, University of Cluj-Napoca, vol. 9, pp. 85-92, 1987.

[20] A. Lupas, "The approximation by means of some positive linear operators," Approximation Theory: (IDOMAT 95 = Proc.International Dormund Meeting on Approximation Theory 1995, pp. 201-229, 1995.

[21] G. Nowak and V. Gupta, "The rate of pointwise approximation of positive linear operators based on q-integer,” Ukrainian Mathematical Journal, vol. 63, no. 3, pp. 403-415, 2011. 
[22] R. Paltanea, Approximation theory using positive linear operators. Boston, Basel, Berlin: Birkhauser, 2004.

[23] G. Philips, "On generalized bernstein polynomials," in Numerical Analysis, D. Griffits and G. Watson, Eds., 1996, pp. 263-269.

[24] D. Stancu, "Approximation of functions by a new class of linear positive operators," Rev.Roum.Math.Pures et Appl., vol. 13, pp. 1173-1194, 1968.

[25] D. Stancu, "Approximation of functions by means of some new classes of positive linear operators," in Numerische Methoden der Approximationstheorie, Proc.Conf.Oberwolfach 1971. Basel: Birkhauser Verlag, 1972, pp. 187-203.

\section{Author's address}

\section{Voichiţa Adriana Radu}

Babes-Bolyai University, FSEGA, Department of Statistics-ForecastsMathematics, Str. Teodor Mihali, No.58-60, RO-400591 Cluj Napoca, Romania

E-mail address: voichita.radu@econ.ubbcluj.ro, voichita.radu@gmail.com 